\title{
1,2-Thio Group Migration in Rh(II) Carbene Reactions
}

\author{
Feng Xu, Weifeng Shi and Jianbo Wang* \\ Key Laboratory of Bioorganic Chemistry and Molecular Engineering of Ministry of Education, \\ Department of Chemical Biology, College of Chemistry, Peking University, Beijing 100871, China \\ wangjb@pku.edu.cn
}

\section{CONTENTS}

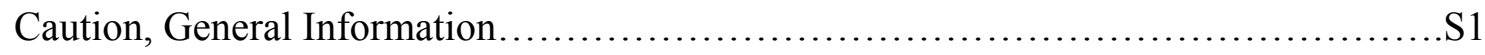



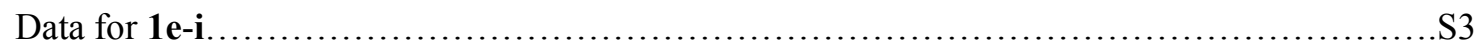

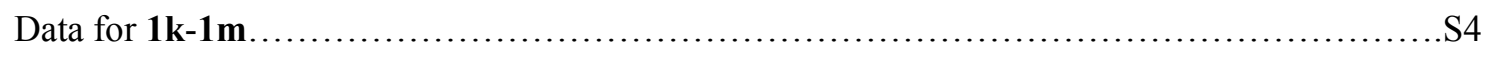

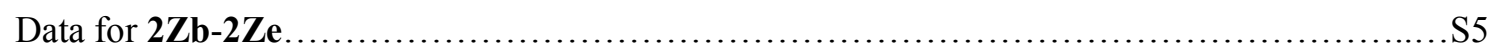

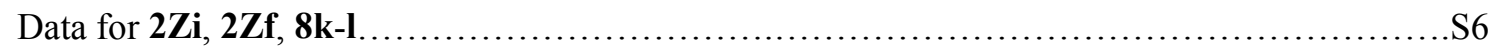






\section{Experimental Section}

Caution: Diazo compounds are potentially explosive. They should be handled with care in a well-ventilated fumehood.

General Information. All reactions with air- and moisture-sensitive components were performed under a nitrogen atmosphere in a flame-dried reaction flask. All solvents were distilled prior to use. $\mathrm{CH}_{2} \mathrm{Cl}_{2}$ was freshly distilled from $\mathrm{CaH}_{2}$ and THF was freshly distilled from $\mathrm{Na}$ before use. For chromatography, 200-300 mesh silica gel was employed.

Ethyl 2-Diazo-3-(benzylthio)pentanoate (1b): $3 \mathrm{~h}, 81 \%$. TLC petroleum ether : ethyl acetate $=30: 1 R_{f}=0.41 ;$ IR (film) 2086, 1686, 1285, 1110, 1070, $701 \mathrm{~cm}^{-1} ;{ }^{1} \mathrm{H}$ NMR (200 $\left.\mathrm{MHz}, \mathrm{CDCl}_{3}\right) \delta 0.95(\mathrm{t}, J=7.6 \mathrm{~Hz}, 3 \mathrm{H}), 1.25(\mathrm{t}, J=7.2 \mathrm{~Hz}, 3 \mathrm{H}), 1.60-1.74(\mathrm{~m}, 2 \mathrm{H}), 3.65(\mathrm{dd}$, $J=6.6,8.2 \mathrm{~Hz}, 1 \mathrm{H}), 3.74(\mathrm{~s}, 2 \mathrm{H}), 4.20(\mathrm{q}, J=7.2 \mathrm{~Hz}, 2 \mathrm{H}), 7.24-7.32(\mathrm{~m}, 5 \mathrm{H}) ;{ }^{13} \mathrm{C} \mathrm{NMR}(50$ $\left.\mathrm{MHz}, \mathrm{CDCl}_{3}\right) \delta 11.7,14.39,26.9,35.8,41.6,60.8,126.9,128.4,128.6,138.1,166.3$; EI-MS $\left(\mathrm{m} / \mathrm{z}\right.$, relative intensity) $250\left[(\mathrm{M}-28)^{+}, 3.90\right], 204(14), 159(8), 91(100), 83(37), 69(42), 57$ (83). Anal. calcd for $\mathrm{C}_{14} \mathrm{H}_{18} \mathrm{~N}_{2} \mathrm{O}_{2} \mathrm{~S}: \mathrm{C}, 60.41 ; \mathrm{H}, 6.52 ; \mathrm{N}, 10.06$. Found: $\mathrm{C}, 60.54 ; \mathrm{H}, 6.54 ; \mathrm{N}$, 10.25 .

Ethyl 2-Diazo-3-(phenylthio)nonanoate (1c): $8 \mathrm{~h}, 55 \%$. TLC petroleum ether : ethyl acetate $=30: 1 R_{f}=0.51$; IR (film) 2085, 1692, 1290, 1121, 1095, 743, $692 \mathrm{~cm}^{-1} ;{ }^{1} \mathrm{H} \mathrm{NMR}$ $\left(200 \mathrm{MHz}, \mathrm{CDCl}_{3}\right) \delta 0.87(\mathrm{t}, J=6.3 \mathrm{~Hz}, 3 \mathrm{H}), 1.11-1.45(\mathrm{~m}, 11 \mathrm{H}), 1.64-1.79(\mathrm{~m}, 2 \mathrm{H})$, 4.05-4.14 (m, 3H), 7.22-7.47 (m, 5H); $\left.{ }^{13} \mathrm{C} \mathrm{NMR} \mathrm{(50} \mathrm{MHz,} \mathrm{CDCl}_{3}\right) \delta 13.96,14.36,22.47$, $27.20,28.65,31.52,33.22,43.79,60.84,127.83,128.85,133.17,133.41$; EI-MS $(\mathrm{m} / \mathrm{z}$, relative intensity) $292\left[(\mathrm{M}-28)^{+}, 25\right], 246$ (41), 211 (17), 189 (36), 155 (38), 109 (100), 95 (29), 67 (53). Anal. calcd for $\mathrm{C}_{17} \mathrm{H}_{24} \mathrm{~N}_{2} \mathrm{O}_{2} \mathrm{~S}$ : C, 63.72; H, 7.55; N, 8.74. Found: C, 64.07; H, 7.45; N, 8.46.

Ethyl 2-Diazo-3-(benzylthio)nonanoate (1d): 2 h, $65 \%$. TLC petroleum ether : ethyl acetate $=30: 1 R_{f}=0.56$; IR (film) 2086, 1689, 1288, 1095, $701 \mathrm{~cm}^{-1} ;{ }^{1} \mathrm{H}$ NMR $(300 \mathrm{MHz}$, $\left.\mathrm{CDCl}_{3}\right) \delta 0.85(\mathrm{t}, J=6.6 \mathrm{~Hz}, 3 \mathrm{H}), 1.23-1.34(\mathrm{~m}, 11 \mathrm{H}), 1.55-1.68(\mathrm{~m}, 2 \mathrm{H}), 3.69-3.74(\mathrm{~m}, 3 \mathrm{H})$, 4.19 (q, $J=7.2 \mathrm{~Hz}, 2 \mathrm{H}), 7.24-7.34(\mathrm{~m}, 5 \mathrm{H}) ;{ }^{13} \mathrm{C} \mathrm{NMR}\left(50 \mathrm{MHz}, \mathrm{CDCl}_{3}\right) \delta 13.9,14.3,22.4$, 26.9, 28.5, 31.4, 33.4, 35.8, 39.9, 60.2, 126.8, 128.3, 128.6, 138.1, 166.3; EI-MS ( $\mathrm{m} / \mathrm{z}$, relative intensity) $306\left[(\mathrm{M}-28)^{+}, 3\right], 260$ (11), 169 (12), 109 (14), 91 (100), 65 (10), 41 (14). Anal. calcd for $\mathrm{C}_{18} \mathrm{H}_{28} \mathrm{~N}_{2} \mathrm{O}_{2} \mathrm{~S}$ : C, 64.64; H, 7.84; N, 8.38. Found: C, 64.92; H, 7.73; N, 8.28. 
2-Diazo-3-(phenylthio)-2-hexanone (1e): $4 \mathrm{~h}, 87 \%$. TLC petroleum ether : ethyl acetate = $30: 1 R_{f}=0.11$; IR (film) 2071, 1641, 1323, 1287, 745, $692 \mathrm{~cm}^{-1} ;{ }^{1} \mathrm{H}$ NMR (300 MHz, CDCl $)_{3}$ $\delta 1.03(\mathrm{t}, J=7.2 \mathrm{~Hz}, 3 \mathrm{H}), 1.65-1.89(\mathrm{~m}, 2 \mathrm{H}), 4.29(\mathrm{~s}, 1 \mathrm{H}), 7.28-7.41(\mathrm{~m}, 5 \mathrm{H}) ;{ }^{13} \mathrm{C}$ NMR $(75$ $\left.\mathrm{MHz}, \mathrm{CDCl}_{3}\right) \delta 11.7,25.2,26.2,43.1,127.5,128.8,132.3,133.1,189.2$; EI-MS $(\mathrm{m} / \mathrm{z}$, relative intensity): $234\left(\mathrm{M}^{+}, 0.49\right), 206$ [(M-28) $\left.{ }^{+}, 3\right], 163$ (1), 135 (3), 125 (12), 110 (25), 97 (12), 77 (4), 65 (9), 43 (100). Anal. calcd for $\mathrm{C}_{12} \mathrm{H}_{14} \mathrm{~N}_{2} \mathrm{OS}$ : C, 61.51; H, 6.02; N, 11.96. Found: C, $65.02 ; \mathrm{H}, 5.78 ; \mathrm{N}, 8.95$.

2-Diazo-3-(benzylthio)-2-hexanone (1f): $2 \mathrm{~h}, 82 \%$. TLC petroleum ether : ethyl acetate = $30: 1 R_{f}=0.12$; IR (film) 2072, 1637, 1366, 1287, $703 \mathrm{~cm}^{-1} ;{ }^{1} \mathrm{H}$ NMR $\left(300 \mathrm{MHz}, \mathrm{CDCl}_{3}\right)$ $\delta 0.93(\mathrm{t}, J=7.2 \mathrm{~Hz}, 3 \mathrm{H}), 1.53-1.73(\mathrm{~m}, 2 \mathrm{H}), 2.04(\mathrm{~s}, 3 \mathrm{H}), 3.70(\mathrm{dd}, J=13.7,18.6 \mathrm{~Hz}, 2 \mathrm{H})$, $3.90(\mathrm{~s}, 1 \mathrm{H}), 7.24-7.31(\mathrm{~m}, 5 \mathrm{H}) ;{ }^{13} \mathrm{C}$ NMR $\left(75 \mathrm{MHz} \mathrm{CDCl}_{3}\right) \delta 11.5,25.3,26.4,36.0,40.7$, 126.8, 128.2, 128.3, 138.1, 189.2; EI-MS ( $/ z / z$, relative intensity) $220\left[(\mathrm{M}-28)^{+}, 5\right], 177(3)$, 129 (17), 97 (6), 91 (100). Anal. calcd for $\mathrm{C}_{13} \mathrm{H}_{16} \mathrm{~N}_{2} \mathrm{OS}$ : C, 62.87; H, 6.49; N, 11.28. Found: C, $62.82 ; \mathrm{H}, 6.60 ; \mathrm{N}, 11.10$.

2-Diazo-3-(phenylthio)-1-phenyl-2-pentanone (1g): $4 \mathrm{~h}, 68 \%$. TLC petroleum ether : ethyl acetate $=30: 1 R_{f}=0.18$; IR (film) 2073, 1607, 1348, 1327, 755, $697 \mathrm{~cm}^{-1} ;{ }^{1} \mathrm{H}$ NMR $\left(200 \mathrm{MHz}, \mathrm{CDCl}_{3}\right) \delta 1.11(\mathrm{t}, J=7.4 \mathrm{~Hz}, 3 \mathrm{H}), 1.72-1.97(\mathrm{~m}, 2 \mathrm{H}), 4.49(\mathrm{t}, J=7.3 \mathrm{~Hz}, 1 \mathrm{H})$, 7.23-7.48 (m, 10H); $\left.{ }^{13} \mathrm{C} \mathrm{NMR} \mathrm{(50} \mathrm{MHz,} \mathrm{CDCl}_{3}\right) \delta 11.8,26.2,44.5,126.7,127.7,128.4,128.9$, 131.3, 132.8, 133.0, 137.3; EI-MS ( $m / z$, relative intensity) $268\left[(\mathrm{M}-28)^{+}, 22\right], 159$ (82), 131 (87), 105 (100), 91 (42), 77 (61). Anal. calcd for $\mathrm{C}_{17} \mathrm{H}_{16} \mathrm{~N}_{2} \mathrm{OS}$ : C, 68.89; H, 5.44; N, 9.45. Found: C, 69.19; H, 5.58; N, 9.13.

2-Diazo-3-(benzylthio)-1-phenyl-2-pentanone (1h): 4.5 h, 65 \%. TLC petroleum ether : ethyl acetate $=30: 1 R_{f}=0.26$; IR (film) $2071,1604,1334,713,697 \mathrm{~cm}^{-1} ;{ }^{1} \mathrm{H}$ NMR (300 $\left.\mathrm{MHz} \mathrm{CDCl}_{3}\right) \delta 1.01(\mathrm{t}, J=7.5 \mathrm{~Hz}, 3 \mathrm{H}), 1.63-1.82(\mathrm{~m}, 2 \mathrm{H}), 3.78(\mathrm{dd}, J=13.8,20.1 \mathrm{~Hz}, 2 \mathrm{H})$, $4.12(\mathrm{t}, J=7.2 \mathrm{~Hz}, 1 \mathrm{H}), 7.18-7.51(\mathrm{~m}, 10 \mathrm{H}) ;{ }^{13} \mathrm{C} \mathrm{NMR}\left(50 \mathrm{MHz}, \mathrm{CDCl}_{3}\right) \delta 11.7,26.5,36.1$, $41.8,126.9,127.6,128.4,128.5,129.0,131.5,137.4,138.1,187.8$; EI-MS $(\mathrm{m} / z$, relative intensity) $282\left[(\mathrm{M}-28)^{+}, 20\right], 253$ (6), 191 (75), 147 (19), 131 (59), 105 (78), 91 (100), 77 (55). Anal. calcd for $\mathrm{C}_{18} \mathrm{H}_{18} \mathrm{~N}_{2} \mathrm{OS}$ : C, 69.65; H, 5.84; N, 9.02. Found: C, 69.77; H, 5.76; N, 9.01 .

Ethyl 2-Diazo-3-(naphthylthio)pentanoate (1i): 6 h, 57 \%. TLC petroleum ether : ethyl 
acetate $=30: 1 R_{f}=0.46$; IR (film) 2086, 1690, 1286, 1116, 813, $744 \mathrm{~cm}^{-1} ;{ }^{1} \mathrm{H}$ NMR (200 $\left.\mathrm{MHz}, \mathrm{CDCl}_{3}\right) \delta 1.03-1.12(\mathrm{~m}, 6 \mathrm{H}), 1.72-1.90(\mathrm{~m}, 2 \mathrm{H}), 4.00-4.18(\mathrm{~m}, 3 \mathrm{H}), 7.43-7.53(\mathrm{~m}, 3 \mathrm{H})$, 7.73-7.91 (m, 4H); ${ }^{13} \mathrm{C}$ NMR $\left(50 \mathrm{MHz}, \mathrm{CDCl}_{3}\right) \delta 11.9,14.2,26.6,45.1,60.8,126.2,126.4$, $127.4,127.5,128.3,129.9,130.6,131.9,132.4,133.5,166.0$; EI-MS ( $m / z$, relative intensity) $286\left[(\mathrm{M}-28)^{+}, 6\right], 257$ (4), 240 (14), 179 (15), 160 (98), 159 (32), 155 (49), 127 (36), 115 (100), 99 (65). Anal. calcd for $\mathrm{C}_{17} \mathrm{H}_{18} \mathrm{~N}_{2} \mathrm{O}_{2} \mathrm{~S}$ : C, 64.94; H, 5.77; N, 8.91. Found: C, 65.02; H, $5.78 ; \mathrm{N}, 8.95$.

Ethyl 2-Diazo-3-phenyl-3-(benzylthio)propionate (1k): $68 \%$. TLC petroleum ether : ethyl acetate $=30: 1 R_{f}=0.36$; IR (film) 2087, 1688, 1243, 1098, $704 \mathrm{~cm}^{-1} ;{ }^{1} \mathrm{H}$ NMR (300 $\left.\mathrm{MHz}, \mathrm{CDCl}_{3}\right) \delta 1.24(\mathrm{t}, J=7.2 \mathrm{~Hz}, 3 \mathrm{H}), 3.72(\mathrm{dd}, J=13.5,18.3 \mathrm{~Hz}, 2 \mathrm{H}), 4.20(\mathrm{q}, J=7.2 \mathrm{~Hz}$, $2 \mathrm{H}), 4.86(\mathrm{~s}, 1 \mathrm{H}), 7.25-7.36(\mathrm{~m}, 10 \mathrm{H}) ;{ }^{13} \mathrm{C} \mathrm{NMR}\left(75 \mathrm{MHz}, \mathrm{CDCl}_{3}\right) \delta 14.3,36.7,42.9,61.0$, $127.0,127.1,127.9,128.4,128.7,128.7,137.3,138.3,165.5$; EI-MS ( $\mathrm{m} / \mathrm{z}$, relative intensity) 298 [(M-28) $\left.{ }^{+}, 14\right], 203$ (10), 175 (8), 147 (15), 135 (17), 103 (13), 91 (100), 77 (7), 65 (11), 45 (9), 29 (30). Anal. calcd for $\mathrm{C}_{18} \mathrm{H}_{18} \mathrm{~N}_{2} \mathrm{O}_{2} \mathrm{~S}: \mathrm{C}, 66.23 ; \mathrm{H}, 5.56 ; \mathrm{N}, 8.58$. Found: C, 66.34; H, $5.71 ; \mathrm{N}, 8.44$.

Ethyl 2-Diazo-3-(p-phenyl)phenyl-3-(phenylthio) propionate (11): $40 \%$ \%. TLC petroleum ether : ethyl acetate $=30: 1 R_{f}=0.33$; IR (film) 2087, 1689, 1291, 1240, 1099, 746, $691 \mathrm{~cm}^{-1}$; ${ }^{1} \mathrm{H}$ NMR $\left(300 \mathrm{MHz}, \mathrm{CDCl}_{3}\right) \delta 1.18(\mathrm{t}, J=7.2 \mathrm{~Hz}, 3 \mathrm{H}), 4.10-4.18(\mathrm{~m}, 2 \mathrm{H}), 5.42(\mathrm{~s}$, 1H), 7.24-7.61 (m, 14H); ${ }^{13} \mathrm{C}$ NMR $\left(75 \mathrm{MHz}, \mathrm{CDCl}_{3}\right) \delta 14.3,46.7,61.0,126.9,127.4,127.5$, $127.6,127.7,128.7,128.9,132.1,133.5,137.0,140.2,141.0,165.4$; EI-MS ( $\mathrm{m} / z$, relative intensity) $360\left[(\mathrm{M}-28)^{+}, 100\right], 315$ (8), 287 (43), 251 (27), 223 (30), 211 (33), 197 (12), 178 (54), 165 (19), 152 (16), 125 (11), 110 (13), 69 (14), 51 (7), 29 (26). Anal. calcd for $\mathrm{C}_{23} \mathrm{H}_{20} \mathrm{~N}_{2} \mathrm{O}_{2} \mathrm{~S}$ : C, 71.11; H, 5.19; N, 7.21. Found: C, 71.28; H, 5.20; N, 6.94.

Ethyl 2-Diazo-3-(p-methoxy)phenyl-3-(phenylthio)propionate (1m): 47 \%. TLC petroleum ether : ethyl acetate $=30: 1 R_{f}=0.19$; IR (film) 2087, 1693, 1508, 1250, 1173, 1032, 830, 744, $690 \mathrm{~cm}^{-1}$; ${ }^{1} \mathrm{H}$ NMR $\left(300 \mathrm{MHz}, \mathrm{CDCl}_{3}\right) \delta 1.18(\mathrm{t}, J=7.2 \mathrm{~Hz}, 3 \mathrm{H}), 3.79(\mathrm{~s}, 3 \mathrm{H})$, 4.08-4.16 (m, 2H), $5.34(\mathrm{~s}, 1 \mathrm{H}), 6.87-6.90(\mathrm{~m}, 2 \mathrm{H}), 7.26-7.46(\mathrm{~m}, 7 \mathrm{H}) ;{ }^{13} \mathrm{C} \mathrm{NMR}(75 \mathrm{MHz}$, $\left.\mathrm{CDCl}_{3}\right) \delta 14.3,46.4,55.2,61.0,114.2,127.7,128.4,128.9,130.0,132.1,133.8,159.3$; EI-MS $\left(\mathrm{m} / \mathrm{z}\right.$, relative intensity) $314\left[(\mathrm{M}-28)^{+}, 100\right], 269$ (10), 241 (38), 205 (52), 177 (73), 165 (78), 149 (18), 135 (56), 110 (45). Anal. calcd for $\mathrm{C}_{18} \mathrm{H}_{18} \mathrm{~N}_{2} \mathrm{O}_{3} \mathrm{~S}$ : C, 63.14; H, 5.30; N, 8.18. Found: C, 63.15; H, 5.49; N, 7.95 . 
(Z)-Ethyl 2-(Benzylthio)-2-pentenoate (2Zb): $12 \mathrm{~h}, 41 \%$. TLC petroether : ethyl acetate $=30: 1 R_{f}=0.47$; IR (film) 1712, 1260,1233, 1133, 1044, 753, $699 \mathrm{~cm}^{-1} ;{ }^{1} \mathrm{H}$ NMR $(300 \mathrm{MHz}$, $\left.\mathrm{CDCl}_{3}\right) \delta 0.80(\mathrm{t}, J=7.5 \mathrm{~Hz}, 3 \mathrm{H}), 1.35(\mathrm{t}, J=7.2 \mathrm{~Hz}, 3 \mathrm{H}), 2.11(\mathrm{~m}, 2 \mathrm{H}), 3.92(\mathrm{~s}, 2 \mathrm{H}), 4.27(\mathrm{q}$, $J=7.2 \mathrm{~Hz}, 2 \mathrm{H}), 7.14-7.29(\mathrm{~m}, 6 \mathrm{H}) ;{ }^{13} \mathrm{C} \mathrm{NMR}\left(75 \mathrm{MHz}, \mathrm{CDCl}_{3}\right) \delta 12.4,14.2,23.9,38.1,61.2$, 125.6, 126.8, 128.2, 128.8, 138.1, 154.3, 165.4; EI-MS ( $\mathrm{m} / \mathrm{z}$, relative intensity) $250\left(\mathrm{M}^{+}, 7\right)$, 204 (23), 159 (7), 131 (11), 91 (100). Anal. calcd for $\mathrm{C}_{14} \mathrm{H}_{18} \mathrm{O}_{2} \mathrm{~S}: \mathrm{C}, 67.16$; H, 7.25. Found: C, $66.77 ; \mathrm{H}, 7.25$.

(Z)-Ethyl 2-(Phenylthio)-2-nonenoate (2Zc): $10 \mathrm{~h}, 83 \%$. TLC petroleum ether : ethyl acetate $=30: 1 R_{f}=0.51$; IR (film) $2927,1713,1478,1241,1044,739,690 \mathrm{~cm}^{-1} ;{ }^{1} \mathrm{H}$ NMR $\left(300 \mathrm{MHz}, \mathrm{CDCl}_{3}\right) \delta 0.87(\mathrm{t}, J=6.7 \mathrm{~Hz}, 3 \mathrm{H}), 1.07(\mathrm{t}, J=7.2 \mathrm{~Hz}, 3 \mathrm{H}), 1.27-1.36(\mathrm{~m}, 6 \mathrm{H})$, 1.47-1.52 (m, 2H), 2.49-2.56 (m, 2H), 4.12 (q, $J=7.2 \mathrm{~Hz}, 2 \mathrm{H}), 7.15-7.27(\mathrm{~m}, 5 \mathrm{H}), 7.40$ (t, $J=$ $7.8 \mathrm{~Hz}, 1 \mathrm{H}) ;{ }^{13} \mathrm{C} \mathrm{NMR}\left(75 \mathrm{MHz}, \mathrm{CDCl}_{3}\right) \delta 13.8,13.9,22.4,28.3,28.9,30.7,31.5,61.3,125.8$, 126.5, 128.0, 128.8, 136.0, 153.2, 165.3; EI-MS ( $\mathrm{m} / \mathrm{z}$, relative intensity) $292\left(\mathrm{M}^{+}, 83\right), 246$ (100), 218 (9), 189 (79), 175 (37), 147 (69), 135 (54), 109 (71), 91 (22), 67 (55). Anal. calcd for $\mathrm{C}_{17} \mathrm{H}_{24} \mathrm{O}_{2} \mathrm{~S}$ : C, 69.82; H, 8.27. Found: $\mathrm{C}, 69.85 ; \mathrm{H}, 8.13$.

(Z)-Ethyl 2-(Benzylthio)-2-nonenoate (2Zd): 19 h, $49 \%$. TLC petroleum ether : ethyl acetate $=30: 1 R_{f}=0.53$; IR (film) 2927, 1713, 1240, 1045, $690 \mathrm{~cm}^{-1} ;{ }^{1} \mathrm{H} \mathrm{NMR}(300 \mathrm{MHz}$, $\left.\mathrm{CDCl}_{3}\right) \delta 0.86(\mathrm{t}, J=7.1 \mathrm{~Hz}, 3 \mathrm{H}), 1.17-1.27(\mathrm{~m}, 8 \mathrm{H}), 1.34(\mathrm{t}, J=7.1 \mathrm{~Hz}, 3 \mathrm{H}), 2.15-2.24(\mathrm{~m}$, 2H), 2.22 (s, 2H), 4.19 (q, $J=7.1 \mathrm{~Hz}, 2 \mathrm{H}), 7.14-7.28(\mathrm{~m}, 6 \mathrm{H}) ;{ }^{13} \mathrm{C} \mathrm{NMR}\left(75 \mathrm{MHz}, \mathrm{CDCl}_{3}\right)$ $\delta 13.9,14.2,22.4,28.0,28.8,30.5,31.4,38.1,61.2,126.1,126.8,128.2,128.8,138.1,153.2$, 165.4; EI-MS ( $\mathrm{m} / z$, relative intensity) $306\left(\mathrm{M}^{+}, 55\right), 260$ (83), 215 (59), 169 (84), 141 (63), 107 (22), 91 (100), 71 (46), 65 (45), 29 (37). Anal. calcd for $\mathrm{C}_{18} \mathrm{H}_{26} \mathrm{O}_{2} \mathrm{~S}: \mathrm{C}, 70.54 ; \mathrm{H}, 8.55$. Found: C, 70.76; H, 8.63.

(Z)-3-(Phenylthio)-3-ene-2-hexanone (2Ze): $2 \mathrm{~h}, 72 \%$. TLC petroleum ether : ethyl acetate $=30: 1 R_{f}=0.35$; IR (film) $1687,1592,1478,1356,1209,1024,740,690 \mathrm{~cm}^{-1} ;{ }^{1} \mathrm{H}$ NMR $\left(300 \mathrm{MHz}, \mathrm{CDCl}_{3}\right) \delta 1.11(\mathrm{t}, J=7.5 \mathrm{~Hz}, 3 \mathrm{H}), 2.29(\mathrm{~s}, 3 \mathrm{H}), 2.47 \sim 2.57(\mathrm{~m}, 2 \mathrm{H})$, 7.15-7.29 (m, 6H); ${ }^{13} \mathrm{C} \mathrm{NMR}\left(75 \mathrm{MHz}, \mathrm{CDCl}_{3}\right) \delta$ 12.7, 20.0, 27.3, 125.8, 127.3, 129.0, 133.6, 135.6, 153.1, 197.5; EI-MS (m/z, relative intensity) $206\left(\mathrm{M}^{+}, 99\right), 135$ (59), 129 (72), 121 (89), 109 (40), 91 (35), 85 (71), 43 (100). Anal. calcd for $\mathrm{C}_{12} \mathrm{H}_{14} \mathrm{O}_{2} \mathrm{~S}: \mathrm{C}, 69.86$; H, 6.84. Found: C, $69.62 ; \mathrm{H}, 6.76$. 
(Z)-3-(Benzylthio)-3-ene-2-hexanone (2Zf): $11 \mathrm{~h}, 54 \%$. TLC petroether : ethyl acetate = $30: 1 R_{f}=0.26$; IR (film) 1677, 1454, 1356, 1227, 1210, 767, $700 \mathrm{~cm}^{-1} ;{ }^{1} \mathrm{H} \mathrm{NMR}(300 \mathrm{MHz}$, $\left.\mathrm{CDCl}_{3}\right) \delta 0.85(\mathrm{t}, J=7.5 \mathrm{~Hz}, 3 \mathrm{H}), 2.24-2.34(\mathrm{~m}, 5 \mathrm{H}), 3.85(\mathrm{~s}, 2 \mathrm{H}), 6.95(\mathrm{t}, J=7.1 \mathrm{~Hz}, 1 \mathrm{H})$, 7.15-7.28 (m, 5H); $\left.{ }^{13} \mathrm{C} \mathrm{NMR} \mathrm{(75} \mathrm{MHz,} \mathrm{CDCl}_{3}\right) \delta 12.5,24.2,26.6,37.6,126.8,128.1,128.8$, 135.2, 138.1, 153.7, 196.7; EI-MS ( $m / z$, relative intensity) $220\left(\mathrm{M}^{+}, 16\right), 129(8), 91(100), 85$ (4), 65 (10), 43 (18). Anal. calcd for $\mathrm{C}_{13} \mathrm{H}_{16} \mathrm{O}_{2} \mathrm{~S}$ : C, 70.87; H, 7.32. Found: C, 70.43; H, 7.29.

(Z)-Ethyl 2-(Naphthylthio)-2-pentenoate (2Zi): 3.5 h, $52 \%$. TLC petroleum ether : ethyl acetate $=30: 1 R_{f}=0.59$; IR (film) $1712,1236,1133,1043,813,745 \mathrm{~cm}^{-1} ;{ }^{1} \mathrm{H}$ NMR (300 $\left.\mathrm{MHz}, \mathrm{CDCl}_{3}\right) \delta 1.04(\mathrm{t}, J=7.1 \mathrm{~Hz}, 3 \mathrm{H}), 1.11(\mathrm{t}, J=7.5 \mathrm{~Hz}, 3 \mathrm{H}), 2.52-2.62(\mathrm{~m}, 2 \mathrm{H}), 4.10$ (q, $J$ $=7.2 \mathrm{~Hz}, 2 \mathrm{H}), 7.31-7.51(\mathrm{~m}, 4 \mathrm{H}), 7.65-7.78(\mathrm{~m}, 4 \mathrm{H}) ;{ }^{13} \mathrm{C} \mathrm{NMR}\left(75 \mathrm{MHz}, \mathrm{CDCl}_{3}\right) \delta 12.8$, 13.9, 24.2, 61.4, 125.5, 126.0, 126.1, 126.1, 126.4, 127.0, 127.6, 128.4, 131.6, 133.3, 133.6, 154.8, 165.4; EI-MS ( $\mathrm{m} / \mathrm{z}$, relative intensity) $286\left(\mathrm{M}^{+}, 65\right), 257$ (3), 240 (44), 211 (20), 155 (100), 128 (53), 115 (51). Anal. calcd for $\mathrm{C}_{17} \mathrm{H}_{18} \mathrm{O}_{2} \mathrm{~S}: \mathrm{C}, 71.30 ; \mathrm{H}, 6.34$. Found: C, 71.31; H, 6.44 .

Ethyl 3-(p-Phenyl)phenyl-2-(phenylthio)propionate (8k): $95 \%$. TLC petroleum ether : ethyl acetate $=30: 1 R_{f}=0.33$; IR (film) 1730, 1486, 1257, 1151, 1025, 822, 747, $693 \mathrm{~cm}^{-1}$; ${ }^{1} \mathrm{H}$ NMR $\left(300 \mathrm{MHz}, \mathrm{CDCl}_{3}\right) \delta 1.05(\mathrm{t}, J=7.2 \mathrm{~Hz}, 3 \mathrm{H}), 3.06-3.28$ (AB part of ABX, $J_{\mathrm{AB}}=$ $13.8 \mathrm{~Hz}, 2 \mathrm{H}), 3.91-3.96$ (X part of $\left.\mathrm{ABX}, J_{\mathrm{AX}}=6.3 \mathrm{~Hz}, J_{\mathrm{BX}}=9.3 \mathrm{~Hz}, 1 \mathrm{H}\right), 3.99-4.08(\mathrm{~m}, 2 \mathrm{H})$, 7.25-7.57 (m, 14H); ${ }^{13} \mathrm{C} \mathrm{NMR}\left(75 \mathrm{MHz}, \mathrm{CDCl}_{3}\right) \delta 13.8,37.4,52.0,61.0,126.8,127.0,127.1$, $127.9,128.6,128.8,129.3,133.0,136.6,139.6,140.6,171.4$; EI-MS ( $\mathrm{m} / z$, relative intensity) $362\left(\mathrm{M}^{+}, 8\right), 289$ (2), 252 (56), 224 (5), 207 (27), 180 (24), 167 (100), 152 (15), 135 (10), 126 (11), 110 (9), 89 (7), 78 (21), 69 (13), 43(16), 29 (13). Anal. calcd for $\mathrm{C}_{23} \mathrm{H}_{22} \mathrm{O}_{2} \mathrm{~S}: \mathrm{C}, 76.21 ; \mathrm{H}$, 6.12. Found: C, 76.22; H, 6.20 .

Ethyl 3-(Phenyl)-2-(benzylthio)propionate (81): $87 \%$. TLC petroleum ether : ethyl acetate $=30: 1 R_{f}=0.42$; IR (film) $1727,1494,1454,1259,1149,1030,747,698 \mathrm{~cm}^{-1} ;{ }^{1} \mathrm{H}$ $\operatorname{NMR}\left(300 \mathrm{MHz}, \mathrm{CDCl}_{3}\right) \delta 1.17(\mathrm{t}, J=7.2 \mathrm{~Hz}, 3 \mathrm{H}), 2.85-3.19\left(\mathrm{~m}, \mathrm{AB}\right.$ part of ABX, $J_{\mathrm{AB}}=$ $13.8 \mathrm{~Hz}, 2 \mathrm{H}$ ), 3.39-3.44 (X part of ABX, $\left.J_{\mathrm{AX}}=6.6 \mathrm{~Hz}, J_{\mathrm{BX}}=9.0 \mathrm{~Hz}, 1 \mathrm{H}\right), 3.74-3.85(\mathrm{~m}, 2 \mathrm{H})$, 7.07-7.10 (m, 2H), 7.18-7.35 (m, 8H); ${ }^{13} \mathrm{C}$ NMR (75 MHz, $\left.\mathrm{CDCl}_{3}\right) \delta 14.0,36.0,37.4,47.2$, $60.9,126.5,127.0,128.2,128.3,128.9,137.2,137.8,171.8$; EI-MS ( $\mathrm{m} / \mathrm{z}$, relative intensity) $300\left(\mathrm{M}^{+}, 4\right), 227$ (2), 193 (2), 178 (51), 135 (9), 123 (23), 104 (21), 91 (100), 78 (9), 65 (10), 51 (2), 45 (6), 29 (7). Anal. calcd for $\mathrm{C}_{18} \mathrm{H}_{20} \mathrm{O}_{2} \mathrm{~S}$ : C, 71.96; H, 6.71. Found: C, 71.90; H, 6.73. 
Ethyl 3-(p-nitro)phenyl-3-(phenylthio)propionate (7): $67 \%$. TLC petroleum ether : ethyl acetate $=30: 1 R_{f}=0.15$; IR (film) $1732,1520,1345,1152,856,748,693 \mathrm{~cm}^{-1} ;{ }^{1} \mathrm{H}$ NMR $\left(300 \mathrm{MHz}, \mathrm{CDCl}_{3}\right) \delta 1.18(\mathrm{t}, J=7.3 \mathrm{~Hz}, 3 \mathrm{H}), 2.88-3.07\left(\mathrm{AB}\right.$ part of ABX, $\left.J_{\mathrm{AB}}=15.9 \mathrm{~Hz}, 2 \mathrm{H}\right)$, 4.04-4.12 (m, 2H), 4.65-4.71 (X part of ABX, $\left.J_{\mathrm{AX}}=6.9 \mathrm{~Hz}, J_{\mathrm{BX}}=8.7 \mathrm{~Hz}, 1 \mathrm{H}\right), 7.24-7.29(\mathrm{~m}$, $7 \mathrm{H}), 8.09-8.13(\mathrm{~m}, 2 \mathrm{H}) ;{ }^{13} \mathrm{C} \mathrm{NMR}\left(75 \mathrm{MHz}, \mathrm{CDCl}_{3}\right) \delta 14.0,40.1,48.6,61.0,123.6,124.1$, 128.5, 128.5, 129.0, 132.1, 133.9, 148.3, 170.0; EI-MS ( $\mathrm{m} / \mathrm{z}$, relative intensity) $331\left(\mathrm{M}^{+}, 13\right)$, 221 (26), 193 (51), 176 (100), 149 (47), 130 (49), 110 (35), 102 (36), 29 (54). Anal. calcd for $\mathrm{C}_{17} \mathrm{H}_{17} \mathrm{NO}_{4} \mathrm{~S}$ : C, 61.61; H, 5.17; N, 4.23. Found: C, 61.41; H, 5.21; N 4.37. 